\title{
Targeting gut microbiota and barrier function with prebiotics to alleviate autoimmune manifestations in NOD mice
}

\author{
Camilla H. F. Hansen ${ }^{1} \cdot$ Christian S. Larsen ${ }^{1} \cdot$ Henriette O. Petersson ${ }^{1} \cdot$ Line F. Zachariassen ${ }^{1} \cdot$ Andreas Vegge $^{2}$. \\ Charlotte Lauridsen $^{3} \cdot$ Witold Kot $^{4} \cdot$ Łukasz Krych $^{5} \cdot$ Dennis S. Nielsen ${ }^{5} \cdot$ Axel K. Hansen $^{1}$
}

Received: 11 March 2019 / Accepted: 25 April 2019 /Published online: 28 May 2019

(C) Springer-Verlag GmbH Germany, part of Springer Nature 2019

\begin{abstract}
Aims/hypothesis Adopting a diet containing indigestible fibre compounds such as prebiotics to fuel advantageous bacteria has proven beneficial for alleviating inflammation. The effect of the microbial changes on autoimmunity, however, remains unknown. We studied the effects of prebiotic xylooligosaccharides (XOS) on pancreatic islet and salivary gland inflammation in NOD mice and tested whether these were mediated by the gut microbiota.

Methods Mother and offspring mice were fed an XOS-supplemented diet until diabetes onset or weaning and were compared with a control-fed group. Diabetes incidence was monitored, insulitis and sialadenitis were scored in histological sections from adult mice, and several metabolic and immune variables were analysed in mice before the development of diabetes. Gut barrier function was assessed using an in vivo FITC-dextran permeability test. The importance of XOS-mediated gut microbial changes were evaluated in antibiotic-treated mice fed either XOS or control diet or given a faecal microbiota transplant from test animals. Results Diabetes onset was delayed in the XOS-fed mice, which also had fewer cellular infiltrations in their pancreatic islets and salivary glands. Interestingly, insulitis was most reduced in the XOS-fed groups when the mice were also treated with an antibiotic cocktail. There was no difference in sialadenitis between the dietary groups treated with antibiotics; the mice were protected by microbiota depletion regardless of diet. Faecal microbiota transplantation was not able to transfer protection. No major differences in glucose-insulin regulation, glucagon-like peptide-1, or short-chain fatty acid production were related to the XOS diet. The XOS diet did, however, reduce gut permeability markers in the small and large intestine. This was accompanied by a more anti-inflammatory environment locally and systemically, dominated by a shift from M1 to M2 macrophages, a higher abundance of activated regulatory T cells, and lower levels of induction of natural killer T cells and cytotoxic T cells.

Conclusions/interpretation Prebiotic XOS have microbiota-dependent effects on salivary gland inflammation and microbiotaindependent effects on pancreatic islet pathology that are accompanied by an improved gut barrier that seems able to heighten control of intestinal diabetogenic antigens that have the potential to penetrate the mucosa to activate autoreactive immune responses.
\end{abstract}

Keywords Gut barrier $\cdot$ Gut microbiota $\cdot$ Insulitis $\cdot$ Mucosal immunology $\cdot$ Prebiotics $\cdot$ Sialadenitis $\cdot$ XOS

Electronic supplementary material The online version of this article (https://doi.org/10.1007/s00125-019-4910-5) contains peer-reviewed but unedited supplementary material, which is available to authorised users.

Camilla H. F. Hansen

camfriis@sund.ku.dk

1 Section of Experimental Animal Models, Department of Veterinary and Animal Sciences, Faculty of Health and Medical Sciences, University of Copenhagen, 1871 Frederiksberg, Denmark

2 Novo Nordisk A/S, Maaloev, Denmark
3 Department of Animal Science, Aarhus University, Tjele, Denmark

4 Department of Environmental Science, Aarhus University, Roskilde, Denmark

5 Department of Food Science, Faculty of Science, University of Copenhagen, Frederiksberg, Denmark 


\section{Research in context}

\section{What is already known about this subject?}

- Prebiotics, including xylooligosaccharides, propagate bacteria associated with an anti-diabetogenic profile in mice and have known anti-inflammatory effects

- The gut microbiota regulates autoimmune reactions towards pancreatic beta cells (insulitis) and salivary glands (sialadenitis)

- An impaired intestinal barrier function precedes the onset of type 1 diabetes in humans and rodent models, but methods to alleviate gut permeability have failed to prevent development of diabetes

\section{What is the key question?}

- Do prebiotic xylooligosaccharides reduce autoimmune manifestations in NOD mice and how do the gut microbiota and barrier function contribute to their anti-inflammatory effects?

\section{What are the new findings?}

- Xylooligosaccharides were found to delay diabetes onset and reduce both insulitis and sialadenitis

- Xylooligosaccharides improved the barrier function in both the small and large intestine, induced antiinflammatory immune cells and reduced the abundance of proinflammatory cells

- The effect of xylooligosaccharides on insulitis was shown to be regulated in a microbe-independent manner linked to improved barrier function in the colon, whereas the effect on sialadenitis was regulated by the microbiota in the same manner as small intestinal barrier function

\section{How might this impact on clinical practice in the foreseeable future?}

- Prebiotic supplements should be tested as a new and feasible approach to improve barrier function and reduce autoimmune reactions in individuals predisposed to developing type 1 diabetes or autoimmune sialadenitis (i.e. Sjögren's syndrome)

$\begin{array}{ll}\text { Abbreviations } & \\ \text { BB-DP } & \text { Bio-breeding diabetes-prone } \\ \text { NFE } & \text { Nitrogen-free extract } \\ \text { NK(T) } & \text { Natural killer (T) } \\ \text { PCoA } & \text { Principal coordinates analysis } \\ \text { GLP-1 } & \text { Glucagon-like peptide-1 } \\ \text { qPCR } & \text { Real-time quantitative PCR } \\ \text { SCFA } & \text { Short-chain fatty acids } \\ \text { XOS } & \text { Xylooligosaccharides } \\ \text { ANCOM } & \text { Analysis of composition of microbiomes } \\ \text { PERMANOVA } & \text { Permutational multivariate ANOVA }\end{array}$

\section{Introduction}

The gut microbiota has become a field of extensive research, and increasing evidence demonstrates the extent to which the bacteria colonising our gut contribute to the development of immune-mediated and metabolic disorders $[1,2]$. Means of effective alleviation of dysbiosis are much needed to help prevent and understand such diseases; xylooligosaccharides
(XOS) are interesting in this regard. XOS pass undigested through the gastrointestinal tract until they reach the ascending colon, where the saccharide molecules selectively propagate residing gut bacteria, including bifidobacteria and lactobacilli $[3,4]$. Both bifidobacteria and lactobacilli have, in rodent models, been used successfully to induce an antiinflammatory response and reduce the incidence of diabetes $[5,6]$.

Antibiotic treatments have also been shown to modify the incidence of type 1 diabetes in NOD mice and bio-breeding diabetes-prone (BB-DP) rats. Depending on age, the spectrum and combination of antibiotics used and the microbes that remain in the gut, antibiotic treatments have been shown to both reduce and accelerate diabetes onset [7-11]. Thus, it is clear that there are both protective and diabetogenic bacteria regulating autoimmune diabetes. Besides modulating the composition of the gut microbiota, dietary fibre is fermentable in the large intestine and forms an important source of shortchain fatty acids (SCFA) with a nutritional role for the epithelial cells lining the gut barrier [12]. Some polysaccharides, such as pectin and xylan, are associated with higher levels of proinflammatory and stress-related transcripts in the colon and have diabetogenic potential in NOD mice [12], whereas 
human milk oligosaccharides, for example, have a tolerogenic effect on the immune system and reduce diabetes incidence in NOD mice [13]. Toivonen et al [12] explain that differences in fibre digestion result in different effects on the microbiota and variation in outcome for the host. The role of the gut microbiota has not been addressed in these studies, however. XOS also shows immunomodulatory effects that seem related to microbial regulation $[3,14]$. Because of the proposed antiinflammatory effects, we wanted to investigate whether XOS would protect against autoimmune destruction of beta cells and salivary glands and whether the protection was dependent on gut microbial changes.

\section{Methods}

The Animal Experiments Inspectorate under the Ministry of Environment and Food approved the study according to the principles of EU Directive 2010/63/EU and the Danish Animal Experimentation Act (number 474 15/05/2014). The studies were performed in accordance with the Guide for the Care and Use of Laboratory Animals of the National Research Council.

Experimental setup Dams of the strain NOD/MrkTac from Taconic (Germantown, NY, USA) were housed at the barrier-protected animal facility, Faculty of Health and Medical Sciences, University of Copenhagen, under standard conditions and in open cages without filter lids. Housing and health monitoring was conducted according to the Federation of European Laboratory Animal Science Associations (FELASA) guidelines [15]. Mice were fed, ad libitum, a modified 1430 Altromin natural diet (Lage, Germany) supplemented with either XOS (Shandong Longlive, Qingdao, China) or standard corn starch as control to balance the level of polysaccharides in the diets in amounts equal to $10 \%$ of the carbohydrate fraction. The experiments were divided into four study set ups, as outlined in electronic supplementary material (ESM) Methods and ESM Fig. 1.

Histology Insulitis and sialadenitis were scored in a blinded fashion on at least 50 islets per pancreas and in 8 sequential sections of each salivary gland from 13-week-old mice on H\&E-stained $5 \mu \mathrm{m}$ sections with $40 \mu \mathrm{m}$ intervals; they were graded as previously described [16].

Gut microbiota composition Faeces were sampled from 4week-old untreated and antibiotic-treated female NOD mice on the control or XOS-supplemented diet as well as 7-weekold NOD mouse recipients of faecal microbiota transplants from control and XOS-fed NOD mice. DNA extraction, tagencoded 16S rRNA gene amplicon MiSeq-based sequencing and sequencing analysis are described in detail in ESM
Methods as well as the real-time quantitative PCR (qPCR) of bacterial DNA from faeces and the mesenteric lymph node.

Metabolic variables For OGTT, 8-week-old mice were fasted for $6 \mathrm{~h}$ prior to oral gavage of glucose, $75 \mathrm{mg} /$ mouse (Amgros, Copenhagen, Denmark); blood glucose was measured at 0 , $15,30,60,90$ and 120 min post glucose challenge. Blood glucose was measured from the lateral tail vein using a glucometer (Abbott Diabetes Care, Copenhagen, Denmark).

Serum from 8-week-old mice fasted for $6 \mathrm{~h}$ was analysed for circulating insulin and leptin levels using the Mouse Metabolic Kit (Meso-Scale Discovery, Rockville, MD, USA) according to the manufacturer's instructions. Samples were read using the Sector Imager 6000 (Meso-Scale Discovery).

SCFA analysis Colon content was sampled from 13-week-old mice and stored on dry ice at $-80^{\circ} \mathrm{C}$ until analysis. SCFA concentration was measured by gas chromatography to assess bacterial fermentation and metabolic activity of the gut microbiota, as previously described [17].

Glucagon-like peptide-1 analysis Serum samples were collected from 13-week-old mice to analyse the total level of glucagon-like peptide-1 (GLP-1), capturing both intact GLP1(7-37) and truncated GLP-1(9-37), using a luminescence oxygen-channelling immunoassay (LOCI) $[18,19]$. The specific assay run had a lower limit of quantification of $30 \mathrm{pmol} / 1$ and an upper limit of $2000 \mathrm{pmol} / \mathrm{l}$.

Intestinal permeability An in vivo intestinal permeability assay was made after 7-week-old mice were fasted and water deprived for $4 \mathrm{~h}$ and subsequently dosed by oral gavage with $600 \mathrm{mg} / \mathrm{kg}$ FITC-dextran (4 kDa; Sigma-Aldrich, St Louis, MO, USA). After $2 \mathrm{~h}$, mice were anaesthetised with fentanyl/fluanisone (Hypnorm, VetaPharma, Leeds, UK) and midazolam (Roche, Hvidovre, Denmark) for retro-orbital blood sampling. The FITC-dextran plasma concentration was analysed in triplicate using a spectrophotometer Spektra MAX Gemini XS (Molecular Devices, Sunnyvale, CA, USA) with excitation $\lambda 485 \mathrm{~nm}$ and emission $\lambda 535 \mathrm{~nm}$.

Zonulin concentration in serum collected from 13-weekold mice was measured with Zonulin BioAssay ELISA Kit (mouse) (United States Biological, Salem, MA, USA) according to the manufacturer's description.

Gene expression by quantitative PCR Immediately after the 13-week-old mice were killed, a $1 \mathrm{~cm}$ fragment of colon and ileum was placed in RNAlater (Ambion, Austin, TX, USA). Homogenisation, RNA isolation with MagMAX-96 RNA Isolation Kit (Ambion) and cDNA synthesis using the HighCapacity cDNA Reverse Transcriptase Kit (Applied Biosystems, Foster City, CA, USA) were performed as 
described previously [3]. Muc1, Muc2, Ocln and Tjp1 were used to assess gut barrier and $\operatorname{Arg} 1, \operatorname{Tg} f \beta$ (also known as Tgfb1), Gzmb, Fasl and Cd8a TaqMan gene expression assays (Applied Biosystem) for macrophage [20] and regulatory and cytotoxic T cell-related genes were used for qPCR analysis. Data were analysed as described previously [3]. Statistical comparison was done on $\Delta \mathrm{C}_{\mathrm{t}}$ values.

Flow cytometry Mice were killed at 13 weeks old and the organs immediately placed on ice. Antibody cocktails were used for staining natural killer T (NKT) cells, cytotoxic T cells, macrophages and regulatory $\mathrm{T}$ cells with CD3-FITC, NK/NKT-PE, CD $8 \alpha$-APC, CD11c-FITC, F4.80-PerCPCY5.5, CD4-PerCP-CY5.5, forkhead box P3 (FOXP3)-PE and CD69-APC (eBioscience, San Diego, CA, USA) in single-cell suspensions prepared from mesenteric and pancreatic lymph nodes and spleens as previously described [11]. Analysis was performed using an Accuri C6 flow cytometer (Accuri Cytometers, Ann Arbor, MI, USA), with the gating strategy as outlined in ESM Fig. 2.

Statistics GraphPad Prism version 6.03 (GraphPad Software, San Diego, CA, USA) was used for statistical analysis; $p$ values $<0.05$ were considered significant and $p$ values $<0.1$ were considered tendencies. Cumulative diabetes incidence curves were calculated using the Kaplan-Meier estimation, while statistical significance between groups was evaluated by the logrank test. For all other data comparing the two groups of mice on a control or XOS diet throughout life, differences were estimated by an unpaired two-tailed Student's $t$ test, or Mann-Whitney $U$ test if the data did not assume Gaussian distribution. Welch's correction was included in the $t$ test if variances were unequal by the $F$ test. Unless stated otherwise in the figure legends, all data comparing the four groups of mice fed the control or XOS diet until weaning with or without antibiotic treatment were compared by twoway ANOVA with Sidak's corrected multiple comparisons test to compare (factor 1) the two dietary groups within (factor 2 ) either the untreated or the antibiotic-treated group. For microbiota analysis, significant clusters in principal coordinates analysis ( $\mathrm{PCoA}$ ) were estimated with permutational multivariate ANOVA (PERMANOVA), and the differences in taxa abundance and prevalence between the groups were estimated with the statistical framework analysis of composition of microbiomes (ANCOM).

\section{Results}

Dietary XOS regulates autoimmune manifestations in NOD mice Diabetes incidence was significantly reduced after 25 weeks on an XOS-supplemented diet compared with a standard control diet (26\% vs $50 \%$, respectively; Fig. 1a).
The incidence was no longer different at 30 weeks of age but the onset of diabetes was significantly later in XOS-fed mice compared with control-fed mice (Fig. 1c). XOS was therefore concluded to delay diabetes onset rather than prevent development of disease. The pancreas and salivary glands were analysed histologically to assess whether the XOSsupplemented diet reduced the degree of insulitis and sialadenitis. The effect on foci size and numbers in the salivary gland was very pronounced (Fig. 1d), whereas the effect on the pancreas was more modest (Fig. 1e).

Effects of diets on islet and salivary gland inflammation in antibiotic-treated mice To verify our speculation that the effects of XOS were mediated by changes in the gut microbiota, faecal microbiota were sequenced and PCoA showed distinct clusters at 4 weeks of age due to diet (PERMANOVA based on unweighted and weighted UniFrac distance matrices: $p<0.05, R^{2}=0.15$ and $p<0.05, R^{2}=0.18$, respectively; Fig. 2a,b). This separation was mainly driven by Parabacteroides spp., as well as four less abundant $(<2 \%)$ genera, showing a relatively higher abundance in the XOS-fed mice (12\%) compared with control-fed mice (1\%) (Fig. 2e). However, a faecal microbiota transplant from XOSand control-fed mice to pups of antibiotic-treated NOD mice housed in germ-free isolators was not sufficient to transfer a delay in diabetes onset (Fig. 1b) despite the separate clustering of their gut microbiota profiles (ESM Fig. 3).

In addition to the effect XOS had on pancreatic and salivary gland inflammation when fed throughout life, a tendency was also observed for reduced insulitis scores in 13-week-old mice fed an XOS diet only during pregnancy and lactation compared with mice on a continuous control diet (Fig. 1e,f). To test whether microbes were necessary for the observed effects, additional groups of pregnant dams on either the control or XOS diet were given a full antibiotic cocktail in the water to deplete the majority of the gut microbiota until all pups were weaned to a control diet. Antibiotic-treated mice on a control diet developed insulitis fully to the same degree as untreated mice (Fig. 1e,f). Interestingly, the slight reduction in insulitis in mice fed an XOS-supplemented diet only until weaning was even greater in mice that were also given antibiotics (Fig. 1e,f). Male NOD mice developed less insulitis than the female mice on antibiotics, but even less when fed an XOSsupplemented diet (Fig. 1g-h). Microbes did not seem necessary for XOS to reduce insulitis, the effect of which, unexpectedly, was more pronounced when microbes were absent.

When the degree of sialadenitis was evaluated in the same groups of mice, we found that an XOS diet early in life was sufficient to reduce the sialadenitis score later in life (Fig. 1d). Both the control- and the XOS-fed mice treated with antibiotics presented with the same low degree of sialadenitis as the 

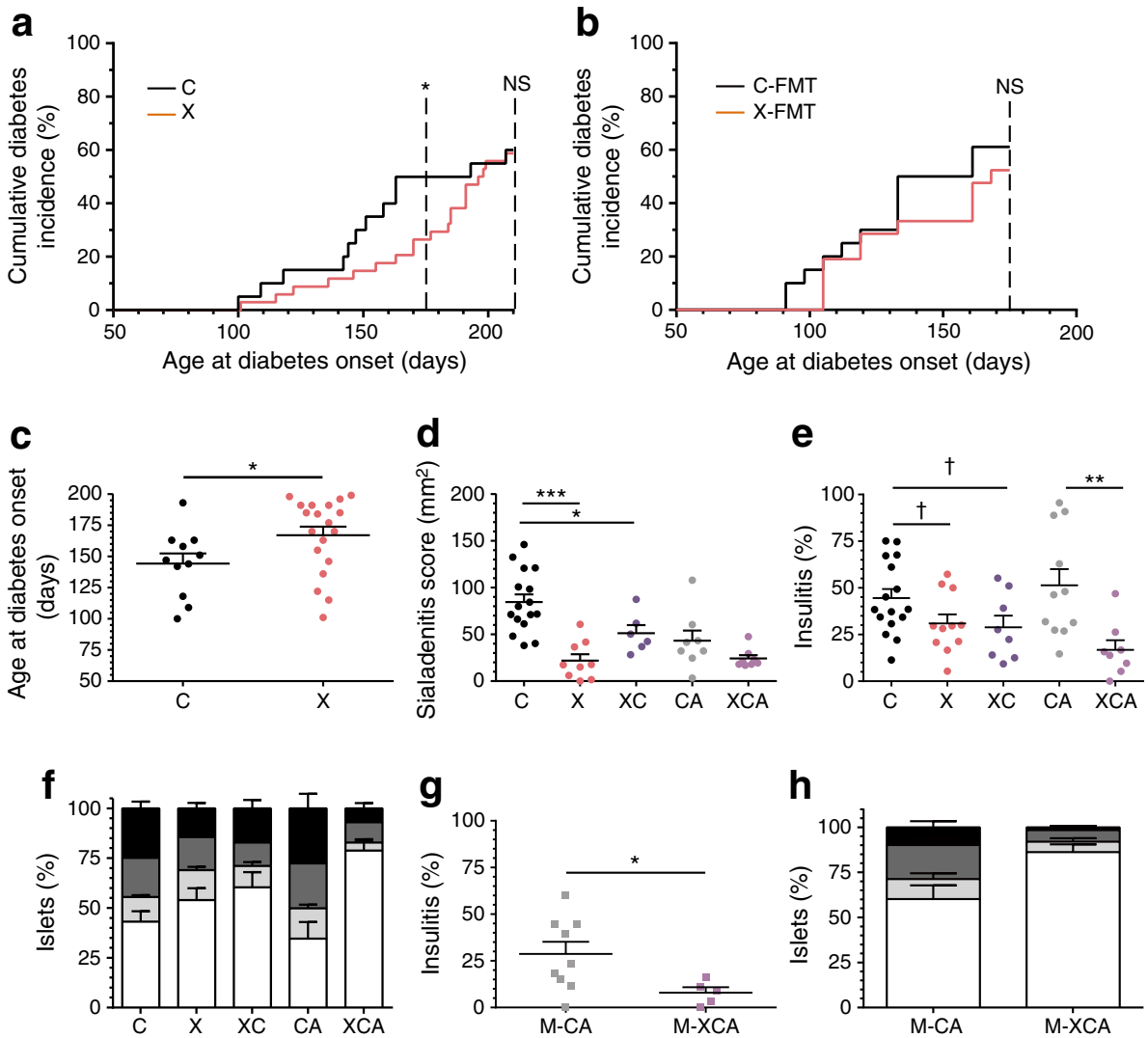

Fig. 1 XOS reduced autoimmune pathologies. (a) Cumulative diabetes incidence in female NOD mice fed a standard chow diet $(C, n=20)$ or an XOS-supplemented diet $(\mathrm{X}, n=34)$ throughout life. (b) Cumulative diabetes incidence in female NOD mice inoculated with faecal microbiota transplants from control-fed (C-FMT, $n=17$ ) and XOS-fed (X-FMT, $n=21$ ) NOD mice. (c) Age at diabetes onset (blood glucose $>12 \mathrm{mmol} / \mathrm{l}$ on two consecutive measurements) was compared for diabetic mice in the two groups shown in (a) during the 30 week observation period. (d, e) Histopathology scores of (d) salivary glands and (e) pancreatic islets. The sialadenitis score and the percentage of islets with insulitis are shown for 13-week-old female NOD mice that had received: a standard chow diet (C, $n=16)$; an XOS-supplemented diet for their whole life (X, $n=9-11)$; an XOS diet until weaning, then switching to the chow diet (XC, $n=6-8)$; and mice treated with antibiotics until weaning and on either control diet (CA, $n=8-11$ ), or XOS diet until weaning and then control diet (XCA, $n=8)$. (f) The percentage of islets with the given scores (see below) for all experimental groups. (g) The percentage of islets with insulitis is shown for 13-week-old male mice treated with antibiotics until weaning and on either control diet (M-CA, $n=9$ ) or XOS diet until weaning and then control diet (M-XCA, $n=5$ ). (h) The percentage of islets with the given scores (see below) for experimental groups in $(\mathbf{g})$. In (f) and (h), white bars, islets with no visible infiltrations; light grey bars, islets with peri-insulitis; dark grey bars, islets with less than $50 \%$ islet infiltration of leucocytes; black bars, islets with more than $50 \%$ islet infiltration. Error bars represent SEM. Comparisons of the survival curves were tested by logrank test after 25 (a, b) and 30 (a) weeks of age, as indicated by vertical dashed lines. Other statistical differences between $\mathrm{C}$ and $\mathrm{X}$ and between M-CA and M-XCA were estimated by an unpaired two-tailed Student's $t$ test or Mann-Whitney $U$ test. The four groups C, XC, CA and XCA were compared by two-way ANOVA with Sidak's corrected multiple comparisons test; $* p<0.05, * * p<0.01$ and $* * * p<0.001 ; p$ values $<0.1$ are indicated by $^{\dagger}$ : in (e), $p=0.07$ for $\mathrm{C}$ vs XC and for $\mathrm{C}$ vs $\mathrm{X}$ untreated XOS-fed mice (Fig. 1d). Hence, no synergistic protective effect was observed for the XOS-fed antibiotic-treated mice as was evident for the development of insulitis.

Antibiotics deplete the gut microbiota The antibiotic cocktail had no effect on the weight of the mice or the amount of water they drank before weaning (ESM Fig. 4a,b). The remaining microbiome of the antibiotic-treated mice was analysed to investigate whether resistant species might remain in the gut and act synergistically with the XOS diet to protect the pancreas. Targeting the 16S rRNA gene (V3 region) with qPCR demonstrated that the overall number of $16 \mathrm{~S}$ copies was reduced more than 4000 fold by the antibiotic cocktail (Fig. 2j).
Amplicon sequencing indicated that the few remaining bacteria in dietary groups consisted primarily of Streptophyta spp., which reached $98 \%( \pm 1 \%)$ of the amplicon abundance (Fig. 2f). Streptophyta spp. have not previously been associated with any immune or disease function. It was furthermore shown that solely one genus at very low abundance, Flavobacterium spp., was significantly elevated in XOS-fed $(0.1 \%$ of the remaining microbiome) compared with controlfed $(0 \%)$ antibiotic-treated mice (Fig. 2g). This single difference in a low-abundance taxon was the main driver for the separate clustering between the two dietary groups in the unweighted PCoA plot: $p<0.05, R^{2}=0.086$ (Fig. 2c). Hence, no difference in the weighted PCoA plot could be observed: $p=$ 

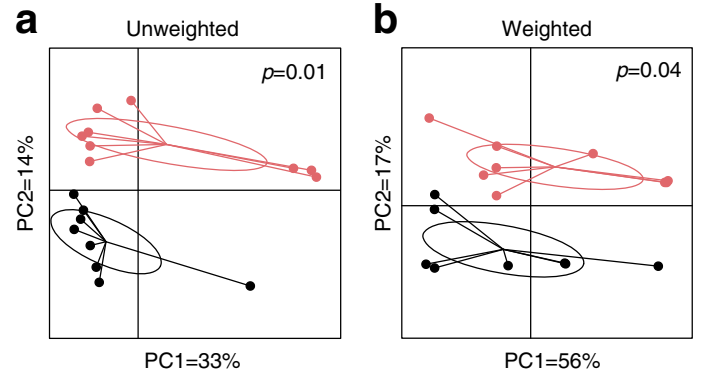

e
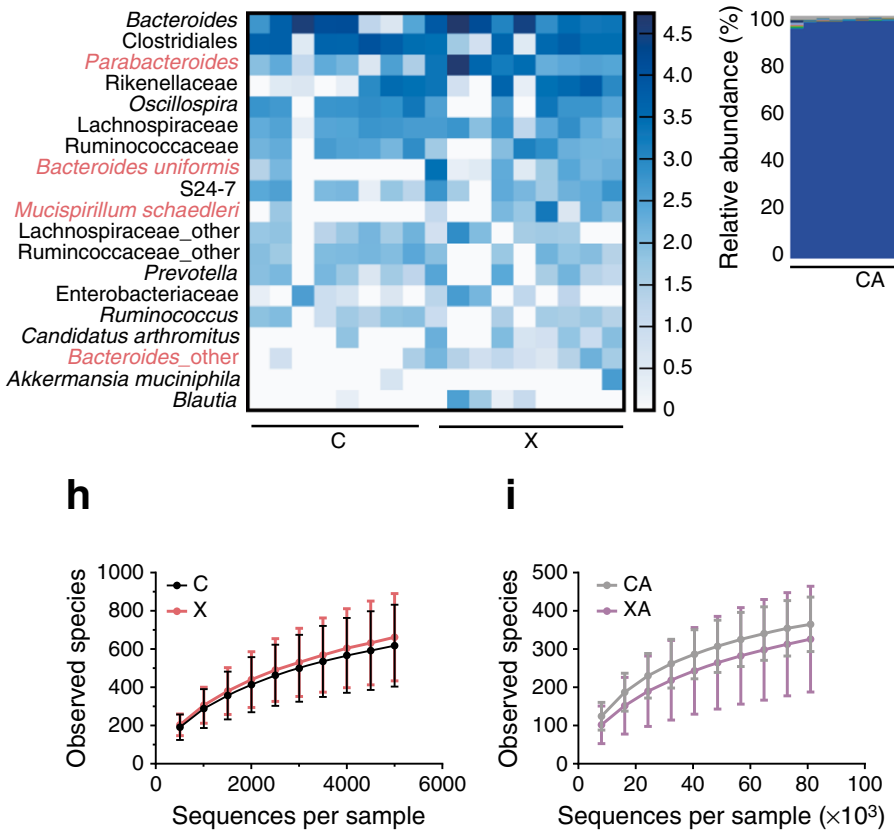

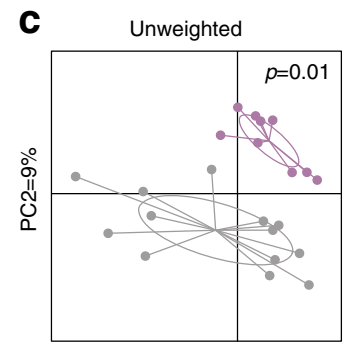

$\mathrm{PC} 1=14 \%$

f
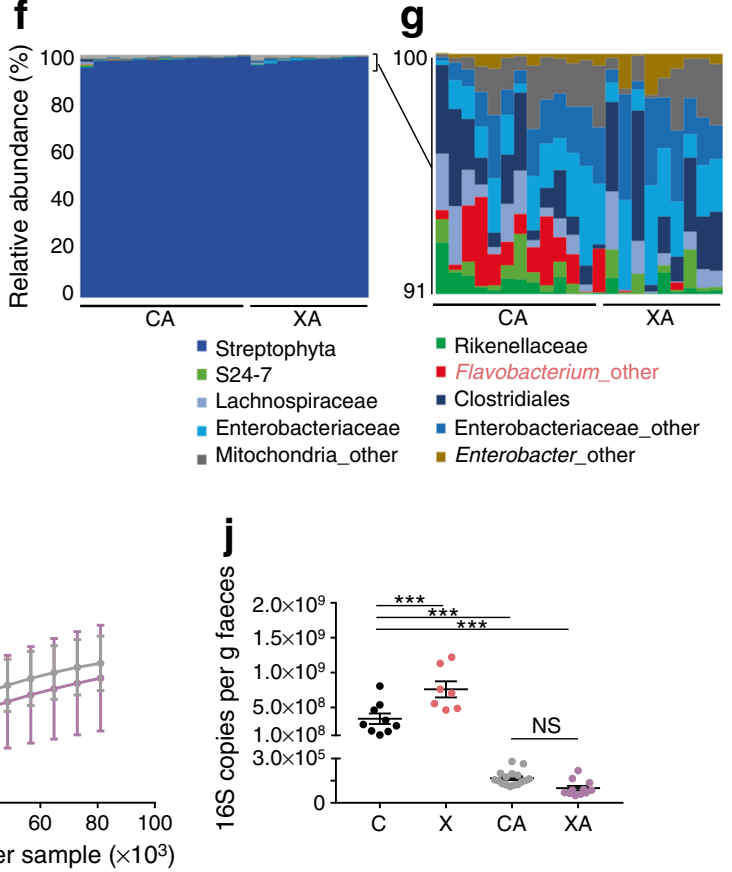

Fig. 2 Effects on the gut microbiota. (a-d) PCoA plots based on unweighted and weighted UniFrac distance matrices depicting data based on 16S rRNA gene tag-encoded amplicon sequencing. The plots illustrate similarities in microbial composition in faecal samples from 4-week-old female NOD mice given untreated water $(\mathbf{a}, \mathbf{b})$ or antibiotic-treated water (c, d) throughout life and fed a standard chow diet (C, black, $n=8$, and CA, grey, $n=13$, respectively), or an XOS-supplemented diet (X, orange, $n=9$, and XA, purple, $n=9$, respectively); PC, principal coordinates. PERMANOVA was used to evaluate group differences. (e) Heatmap demonstrating relative abundance of bacterial taxa in the gut microbiota of untreated control-fed (C, $n=8)$ and XOS-fed (X, $n=9)$ mice. The abundance of taxa in red is significantly different $(\mathrm{ANCOM}<0.05)$ between the two groups. (f, $\mathbf{g})$ Graphs represent the relative abundance of the

$0.17, R^{2}=0.17$ (Fig. 2d) and, thus, collectively, the data strongly suggest that XOS had direct microbiotaindependent effects on insulitis.

Dietary impact on metabolism and glucose tolerance Having a different diet, one based on more long-chained carbohydrates instead of monomer sugar molecules, would naturally be expected to manifest as differences in the host metabolism. Prebiotics have been shown to improve glucose homeostasis and insulin sensitivity by SCFA-mediated activation of intestinal gluconeogenesis [21]. We found no differences in weight remaining taxa in antibiotic-treated control- $(\mathrm{CA}, n=13)$ and XOS-fed (XA, $n=9$ ) mice. Taxa in red have significantly different abundances (ANCOM < 0.05 ) between the dietary groups. Taxa denoted as 'other' indicates ambiguity in the assignment, i.e. more than one taxon could be assigned to this cluster at the given taxonomic level. (h, i) Alpha diversity (observed species index) in the 4-week-old untreated (h; C, $n=8 ; \mathrm{X}, n=9$ ) and antibiotic-treated (i; CA, $n=13$; XA, $n=9$ ) NOD mice. Error bars represent SD. (j) Total number of $16 \mathrm{~S}$ copies quantified by qPCR of the 16S rRNA gene (V3 region) in untreated and antibiotic-treated 4-weekold NOD mice. The $y$-axis is on a base 10 logarithmic scale. Error bars represent SEM. Statistical analysis was done by two-way ANOVA with Tukey's multiple comparisons test to compare all four group means, regardless of diet and antibiotic treatment; $* * * p<0.001$

gain or food intake based on the nitrogen-free extract (NFE) of the diets (ESM Fig. 4c,d), and XOS supplementation had no effect on fasting blood glucose levels, serum leptin and insulin, insulin resistance or GLP-1 (ESM Fig. 5a-f), even though fermentation of XOS slightly increased the production of acetic acid in the colon (ESM Fig. 5g). There was no effect of XOS on $n$-butyric acid or propionic acid (ESM Fig. 5h,i). From these findings, we concluded that the effects of XOS on the host were not mediated through any major shifts in metabolism. A gluten-free diet has been shown to have favourable effects in NOD mice [22, 23], but the effect of 

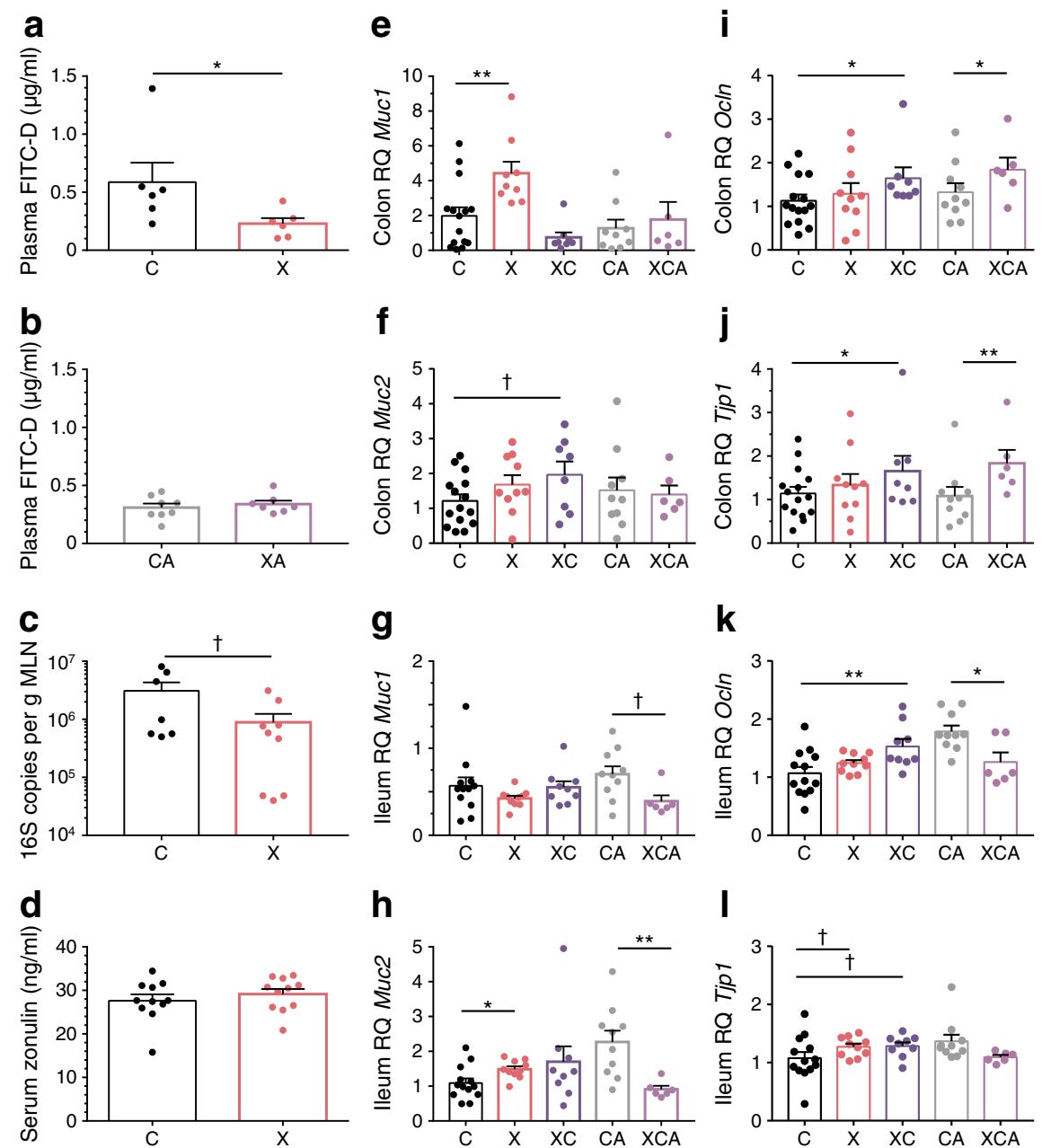

Fig. 3 Microbiota regulated dietary improvement of the gut barrier function. $(\mathbf{a}, \mathbf{b})$ Spectrophotometric analysis of plasma FITC-dextran (FITCD) level $2 \mathrm{~h}$ post oral gavage at 7 weeks of age in (a) mice fed the control $(\mathrm{C}, n=6)$ or XOS (X, $n=6)$ diet for their whole life, and (b) in mice treated with antibiotics for their whole life and fed the control (CA, $n=8)$ or XOS (XA, $n=7$ ) diet. (c) qPCR targeting 16S rRNA gene (bacterial DNA) in the mesenteric lymph nodes (MLN) at 7 weeks of age ( $n=7-9$ /group). The $y$-axis is on a base 10 logarithmic scale. (d) Serum zonulin measured by ELISA at 13 weeks of age ( $n=11$ /group). (e-l) Relative gene expression of $M u c 1(\mathbf{e}, \mathbf{g}), \operatorname{Muc} 2(\mathbf{f}, \mathbf{h}), O c l n(\mathbf{i}, \mathbf{k})$ and $T j p 1(\mathbf{j}, \mathbf{l})$ in colon and ileum biopsies sampled at 13 weeks of age from female NOD mice fed the standard chow diet (C), the XOS-supplemented diet for their whole life

gluten seems, in contrast to the XOS diet, to be mediated by direct regulation of beta cell activity $[24,25]$. We therefore suspected that a gluten-free diet in combination with XOS could provide further protection. However, the incidence of diabetes remained low, at around $20 \%$, on a gluten-free diet regardless of XOS supplementation (ESM Fig. 5j).

Prebiotic improvement of the intestinal barrier function and mucosal inflammation A FITC-dextran assay was used to evaluate the gut integrity as prebiotics have previously been shown to alter gut morphology and function [26]. The barrier was indeed less permeable in mice fed the XOS-supplemented
$(\mathrm{X})$ or only until weaning $(\mathrm{XC})$, and antibiotic-treated mice fed either chow (CA) or the XOS- supplemented diet until weaning (XCA). Error bars represent SEM. Statistical differences between $C$ and $X$ and between CA and XA were estimated by an unpaired two-tailed Student's $t$ test or Mann-Whitney $U$ test. The four groups $\mathrm{C}, \mathrm{XC}, \mathrm{CA}$ and XCA were compared by two-way ANOVA with Sidak's corrected multiple comparisons test. All comparisons of gene expression data were carried out on $\Delta \mathrm{C}_{\mathrm{t}}$ values; ${ }^{*} p<0.05,{ }^{*} p<0.01 . p$ values $<0.1$ are indicated by ${ }^{\dagger}:$ in $(\mathbf{c})$, $p=0.07$ for $\mathrm{X}$ vs $\mathrm{C}$; in (f), $p=0.09$ for $\mathrm{XC}$ vs $\mathrm{C}$; in $(\mathbf{g}), p=0.06$ for $\mathrm{XCA}$ vs $\mathrm{CA}$; and in (l), $p=0.07$ for $\mathrm{X}$ vs $\mathrm{C}$ and $\mathrm{XC}$ vs $\mathrm{C}$. RQ, relative quantification

diet compared with mice fed the control diet (Fig. 3a). This was confirmed with qPCR of the 16S rRNA gene in mesenteric lymph node, which showed that fewer microbes were able to cross the gut barrier and reach the draining lymph node in XOS-fed mice compared with control-fed mice (Fig. 3c). Gene expression of mucus-related genes was also upregulated in mice on the XOS diet in both the ileum (Muc2) and the colon $(M u c 1)$, with the strongest effect in mice that were not switched to a chow diet at weaning (Fig. 3e,h). In contrast, other barrier-related genes such as Ocln and Tjp1 (encoding occludin and tight junction protein 1, respectively) were significantly upregulated in both the ileum and colon of mice fed 
XOS early in life only (Fig. 3i-1). Supplementation with XOS in early life seemed to regulate those parts of the barrier function in a microbiota-independent manner, as the difference persisted in antibiotic-treated mice (Fig. 3i-j). This was, however, only the case for the large intestine, as the FITC-dextran assay, representative of ileum barrier function (Fig. 3b), as well as gene expression in ileal tissue (Fig. 3k,l) gave no such indications of an improved barrier in antibiotic-treated mice. The intestinal tight junction modulator zonulin is upregulated in serum of individuals with type 1 diabetes before the onset of disease [27], but the effect of XOS on intestinal permeability did not seem to be mediated by zonulin as the serum level of the protein was similar between the two dietary groups (Fig. $3 d)$.

\section{Microbiota-mediated dietary changes in mucosal inflamma-}

tion The gut barrier is important for homeostasis of the immune system both within and beyond the mucosal immune populations. Potential anti-inflammatory effects of the XOS diet were therefore analysed by flow cytometry both locally and systemically. Levels of $\mathrm{CD} 8^{+}$NKT cells and cytotoxic $\mathrm{CD}^{+} \mathrm{T}$ cells were lower in XOS-fed mice compared with control-fed mice, both in the pancreatic and mesenteric lymph nodes as well as in the spleen (Fig. 4a,b). This was confirmed by qPCR of $C d 8 a$, Gzmb (encoding granzyme B) and Fasl (encoding Fas ligand), which showed expression of these genes was similarly downregulated in XOS-fed mice, but not in those switched to the control diet at weaning (Fig. 4c-e). In addition, the proportion of activated $\left(\mathrm{CD} 69^{+}\right)$regulatory $\mathrm{T}$ cells was slightly higher in the local lymph nodes of XOS-fed mice compared with control-fed mice (Fig. 4f). The $\operatorname{Tg} f \beta$ gene was expressed at a higher level in mice fed the XOS diet early in life (Fig. 4j), which further indicated a more anti-inflammatory local environment. In addition, $\mathrm{F} 4.80^{+}$macrophages were induced by the XOS diet both locally and systemically (Fig. 4g). However, the proportion of classic $\mathrm{CD} 11 \mathrm{c}^{+} \mathrm{M} 1$ macrophages, which encourage inflammation, was not higher in XOS-fed mice. In fact, this population was decreased in the spleen (Fig. 4h). It is likely that the increased abundance of macrophages was due to an increase in anti-inflammatory M2 macrophages, which are also major producers of TGF- $\beta$. This was also suggested by gene expression of the M2 macrophage marker Argl (encoding arginase 1), which was expressed at higher levels in XOS-fed mice (Fig. 4i).

The function of the dietary-induced changes in immune cells was investigated by adoptive transfer of splenocytes from XOS- and control-fed NOD mice to NOD/SCID mice. The transfer induced diabetes in both groups and, surprisingly, the effect was greater in the XOS group (Fig. 5a). Perhaps the lower proportion of regulatory $\mathrm{T}$ cells evident in the spleen of
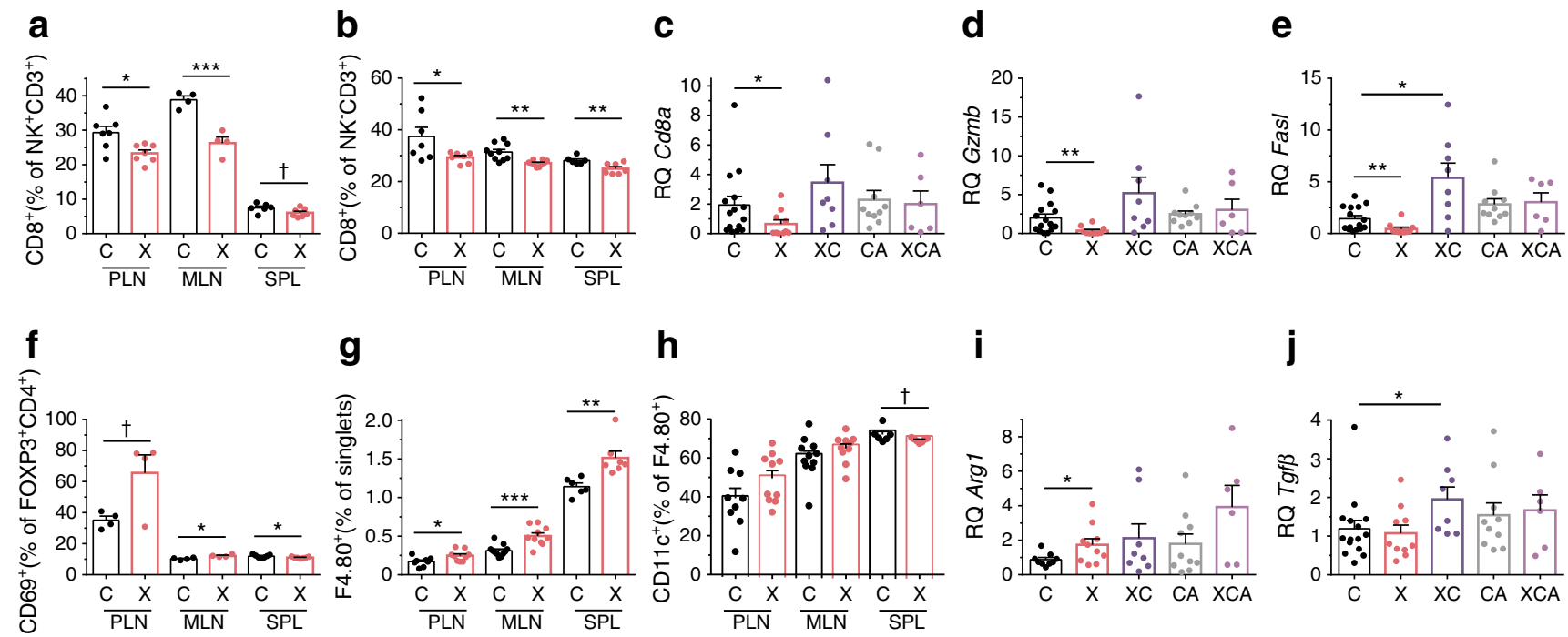

Fig. 4 XOS reduced intestinal inflammation. Flow cytometric analysis of cells isolated from the pancreatic lymph node (PLN), mesenteric lymph node (MLN) and spleen (SPL) in 13-week-old female NOD mice fed control (C) or XOS (X) diet throughout life ( $n=7 /$ group). (a, b) Percentages of (a) $\mathrm{CD}^{+}$NKT cells and (b) cytotoxic $\mathrm{CD} 8^{+} \mathrm{T}$ cells. (ce) Relative gene expression in colon of (c) Cd8a, (d) Gzmb and (e) Fasl. (f-h) Flow cytometric analysis of the percentage of (f) $\mathrm{CD}^{+} 9^{+}$regulatory $\mathrm{T}$ cells ( $n=4 /$ group), (g) F4.80 $0^{+}$macrophages, and (h) CD11 $\mathrm{c}^{+} \mathrm{M} 1 \mathrm{mac}-$ rophages. (i, j) Relative gene expression of the M2 macrophage markers (i) Argl and (j) $T g f \beta$. For gene expression analyses, mice were fed standard chow diet $(\mathrm{C}, n=15)$, or XOS-supplemented diet for their whole life
(X, $n=8)$ or only until weaning (XC, $n=8)$; antibiotic-treated mice were fed either chow (CA, $n=10$ ) or the XOS-supplemented diet (XCA, $n=6$ ) until weaning. Error bars represent SEM. Statistical differences between $\mathrm{C}$ and $\mathrm{X}$ were estimated by an unpaired two-tailed Student's $t$ test or Mann-Whitney $U$ test. The four groups $\mathrm{C}, \mathrm{XC}, \mathrm{CA}$ and XCA were compared by two-way ANOVA with Sidak's corrected multiple comparisons test. All comparisons of gene expression data were carried out on $\Delta \mathrm{C}_{\mathrm{t}}$ values; $* p<0.05, * * p<0.01$ and $* * * p<0.001 . p$ values $<0.1$ are indicated by $^{\dagger}$ : in (a), $p=0.07$ for $\mathrm{X}$ vs $\mathrm{C}$ in spleen; in (f), $p=0.07$ for $\mathrm{X}$ vs $\mathrm{C}$ in pancreatic lymph node; and in (h), $p=0.07$ for $\mathrm{X}$ vs $\mathrm{C}$ in spleen. RQ, relative quantification 

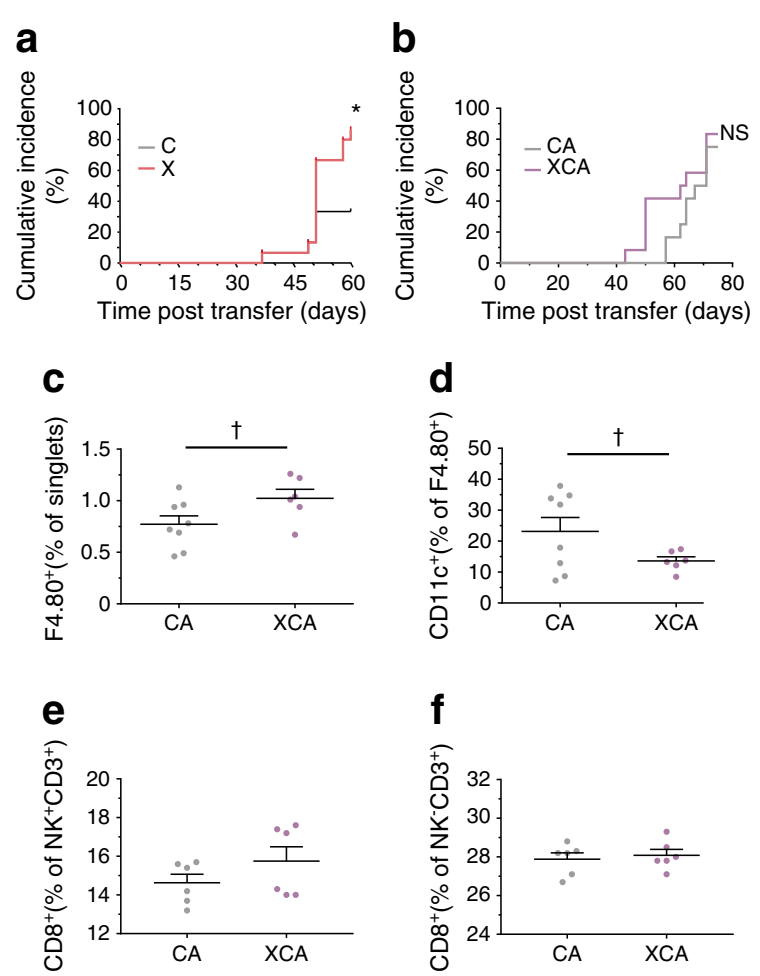

Fig. 5 Adoptive transfer of splenocytes failed to delay diabetes onset. (a, b) Cumulative incidence of diabetes: splenocytes from (a) 13-week-old female NOD mice on the control (C) or XOS (X) diet throughout life or (b) 13-week-old antibiotic-treated mice on the control (CA) diet or XOS diet until weaning (XCA) were adoptively transferred to 8-week-old female NOD/SCID mice ( $n=15 /$ group from mice without antibiotics and $n=12$ /group from mice with antibiotics). Onset of diabetes was monitored by weekly blood glucose measurements for 9-11 weeks post transfer. (cf) Flow cytometric analysis of the splenocytes transferred from the mice treated with antibiotics until weaning demonstrated the percentage of (c) F4.80 $0^{+}$macrophages, (d) $\mathrm{CD} 11 \mathrm{c}^{+} \mathrm{M} 1$ macrophages, (e) $\mathrm{CD}^{+} \mathrm{NKT}$ cells and (f) cytotoxic $\mathrm{CD}^{+}$T cells. Error bars represent SEM. Comparisons of the survival curves were tested by logrank test. Other statistical differences between CA and XCA were estimated by an unpaired two-tailed Student's $t$ test or Mann-Whitney $U$ test; ${ }^{*} p<0.05$. $p$ values $<0.1$ are indicated by ${ }^{\dagger}$ : in (c), $p=0.06$ for XCA vs CA; in (d), $p=0.09$ for XCA vs CA

XOS-fed mice (Fig. 4f) contributed to the accelerated development of diabetes regardless of any potential antiinflammatory effects of other immune cells present. In addition, we transferred splenocytes from XOS- and control-fed NOD mice treated with antibiotics early in life, as the most prominent effect of XOS on insulitis was evident in these groups (Fig. 1e). Nonetheless, the incidence of diabetes was similar between the two groups (Fig. 5b), which corresponded well with the lack of difference in $\mathrm{CD} 8^{+}$immune cells in antibiotic-treated mice (Fig. 5e,f). Hence, the systemic effect of XOS on macrophages seems not to, in either antibiotictreated (Fig. 5c,d) or untreated (Fig. 4g,h) mice, play any important role in the development of diabetes. The explanation for the effects of XOS on diabetes development may lie more in the local environment surrounding the pancreas.

\section{Discussion}

Here, we demonstrate that the prebiotic compound XOS can change the gut microbiota and delay the onset of type 1 diabetes in NOD mice and reduce autoimmune cellular reactions against pancreatic beta cells and salivary glands. This is in line with previous results demonstrating that a variety of intestinal bacteria can regulate the degree of both insulitis and sialadenitis in NOD mice [16, 28, 29]. The high degree of insulitis in antibiotic-treated mice resembles that observed in germ-free NOD mice [29]. The effect is similar to that seen in a previous study using the same broad-spectrum antibiotic cocktail during pregnancy to eradicate the microbiota of the mothers before faecal transplants of a complete microbiota at birth; $100 \%$ of the female offspring developed diabetes [30]. However, the unexpected reduction in insulitis in antibiotictreated mice fed XOS early in life suggested that the alleviating effect is not necessarily a product of microbiota-induced anti-diabetogenic signals. Increasing evidence is establishing direct pathways by which dietary oligosaccharides can act on the immune system: for example, the gut barrier is permeable to milk oligosaccharides during infancy [31], and it can bind specific sugar receptors on human cells $[32,33]$ and thereby modulate the immune response systemically. Of course, the shift in microbial composition towards species with known probiotic potential cannot be excluded from also playing a protective role. There was, for example, a clear increase in Parabacteroides spp. which have been associated with antiinflammatory and diabetes-reducing properties [34, 35]. Nonetheless, faecal microbiota transplantation from XOSfed mice was not sufficient to transfer a reduction in time to diabetes onset. It is worth considering that in such a scenario, without XOS as an available nutrient source, the microbiota may function differently and, therefore, a microbiotamediated anti-inflammatory effect of an XOS-supplemented diet should therefore not be ruled out. In contrast to the effect on insulitis, the development of sialadenitis was clearly regulated by gut bacteria, which is comparable with our previous study in germ-free mice [16]. These findings warrant further investigation into the regulatory role of Parabacteroides spp. in the development of sialadenitis rather than insulitis.

According to the 'perfect storm' hypothesis, an increased intestinal permeability would allow for inappropriate food and microbial antigens to traverse the gut mucosa, triggering an aberrant immune response to otherwise harmless proteins [36]. Fewer $\mathrm{CD} 8^{+} \mathrm{NKT}$ cells and cytotoxic $\mathrm{CD} 8^{+} \mathrm{T}$ cells in the lymphoid tissues and reduced translocation of bacteria to the mesenteric lymph nodes further support the minimal passage of intestinal antigens across the mucosal barrier in XOSfed mice. Functional studies in BB-DP rats demonstrate that increased permeability of the small intestine, but not the colon, appeared before the development of insulitis [37]. A later study verified that BB-DP rats had a higher urinary excretion 
ratio of lactulose:mannitol than Wistar rats before the onset of diabetes [38]. However, no difference was observed in survival or islet inflammation for BB-DP rats when butyrate was administered to strengthen intestinal tight junctions, bringing into question the importance of a leaky gut, at least in the small intestine.

If and how the mucosal inflammation contributes to inducing an autoimmune cascade in the nearby pancreas remain unknown, but the antibiotic-treated mice shed some light on the importance of a leaky gut. The small intestinal barrier in these mice was no longer improved by the XOS diet, in contrast to markers of colonic barrier function. This is in accordance with a study from the $90 \mathrm{~s}$, demonstrating that dietary fibre increased crypt and mucin-containing goblet cell numbers in germ-free rats $[26,39]$ and that colonisation of the gut actually abolished this effect. The discrepancy between the different sections of gut is interesting as the XOS diet was also able to reduce islet inflammation in microbiota-depleted mice, suggesting that the colonic barrier function rather than the small intestinal barrier was involved in the alleviating effect. In support of this, a study demonstrated that glucagon-like peptide- 2 reduced small intestinal transepithelial resistance in NOD mice but failed to delay the onset of type 1 diabetes [40]. In another study, infection with Citrobacter rodentium increased large intestinal permeability at weaning, and this was followed by systemic infection of mesenteric and pancreatic lymph nodes, proliferation of diabetogenic $\mathrm{CD} 8^{+} \mathrm{T}$ cells and accelerated development of insulitis; this was in contrast to infection with a modified strain that did not disrupt the large intestinal barrier [41]. The latter study also showed that the small intestinal permeability was increased at 12 weeks of age, long after islet inflammation began, but not before 12 weeks.

In conclusion, the inconsistency between studies investigating the ability of the gut barrier to regulate the development of diabetes appears to highlight the need to distinguish segments of the gastrointestinal tract based on functional differences. It is important to note that the small intestinal barrier function may play a more profound role in the systemic immune response and autoimmune reactions in the salivary glands, as the XOS diet not only failed to alleviate small intestinal permeability in antibiotic-treated mice, but also had no effect on systemic immunity and sialadenitis in the absence of a fully colonised gut. Functional studies combining methods to alleviate and aggravate gut permeability with environmental factors associated with a modified barrier function, such as the XOS diet, in various sections of the gastrointestinal tract are necessary to address such hypotheses. Nonetheless, considering the previous findings on increased intestinal permeability in individuals with type 1 diabetes [42, 43], eating a prebiotic supplement seems a feasible approach that, because of the effects on mucus production and tight junction proteins, may be able to limit the impact of environmental triggers that might otherwise translocate the compromised gut barrier.

Acknowledgements The authors thank S. Høgh Madsen and S. Halkier from Research Bioanalysis at Novo Nordisk for analytical support.

Data availability The datasets generated and/or analysed during the current study are available from the corresponding author on reasonable request.

Funding This project was funded by the $3 \mathrm{G}$ Center (Gut, Grain \& Greens), supported by the Innovation Fund Denmark.

Duality of interest The authors confirm there is no duality of interest associated with this manuscript.

Contribution statement CSL, HOP, ŁK, AV, WK, LFZ and CL obtained research data. DSN and AKH contributed to the conception and interpretation of this work. CHFH designed the study, obtained research data and wrote the manuscript. All authors revised and approved the final version of the manuscript. CHFH is responsible for the integrity of the work as a whole.

\section{References}

1. Arora T, Backhed F (2016) The gut microbiota and metabolic disease: current understanding and future perspectives. J Intern Med 280(4):339-349. https://doi.org/10.1111/joim.12508

2. Gensollen T, Iyer SS, Kasper DL, Blumberg RS (2016) How colonization by microbiota in early life shapes the immune system. Science 352(6285):539-544. https://doi.org/10.1126/science. aad 9378

3. Hansen CH, Frokiaer H, Christensen AG et al (2013) Dietary xylooligosaccharide downregulates IFN- $\gamma$ and the low-grade inflammatory cytokine IL-1 $\beta$ systemically in mice. J Nutr 143(4): 533-540. https://doi.org/10.3945/jn.112.172361

4. Petersen A, Bergstrom A, Andersen JB et al (2010) Analysis of the intestinal microbiota of oligosaccharide fed mice exhibiting reduced resistance to Salmonella infection. Benef Microbes 1(3): 271-281. https://doi.org/10.3920/BM2010.0016

5. Calcinaro F, Dionisi S, Marinaro M et al (2005) Oral probiotic administration induces interleukin-10 production and prevents spontaneous autoimmune diabetes in the non-obese diabetic mouse. Diabetologia 48(8):1565-1575. https://doi.org/10.1007/s00125005-1831-2

6. de Goffau MC, Luopajarvi K, Knip M et al (2013) Fecal microbiota composition differs between children with beta-cell autoimmunity and those without. Diabetes 62(4):1238-1244. https://doi.org/10. 2337/db12-0526

7. Brown K, Godovannyi A, Ma C et al (2016) Prolonged antibiotic treatment induces a diabetogenic intestinal microbiome that accelerates diabetes in NOD mice. ISME J 10(2):321-332. https://doi. org/10.1038/ismej.2015.114

8. Brugman S, Klatter FA, Visser JTJ et al (2006) Antibiotic treatment partially protects against type 1 diabetes in the Bio-Breeding diabetes-prone rat. Is the gut flora involved in the development of type 1 diabetes? Diabetologia 49(9):2105-2108. https://doi.org/10. 1007/s00125-006-0334-0 
9. Buschard K, Pedersen C, Hansen SV, Hageman I, Aaen K, Bendtzen K (1992) Anti-diabetogenic effect of fusidic acid in diabetes prone BB rats. Autoimmunity 14(2):101-104. https://doi.org/ 10.3109/08916939209083127

10. Candon S, Perez-Arroyo A, Marquet C et al (2015) Antibiotics in early life alter the gut microbiome and increase disease incidence in a spontaneous mouse model of autoimmune insulin-dependent diabetes. PLoS One 10(5):e0125448. https://doi.org/10.1371/journal. pone. 0125448

11. Hansen CH, Krych L, Nielsen DS et al (2012) Early life treatment with vancomycin propagates Akkermansia muciniphila and reduces diabetes incidence in the NOD mouse. Diabetologia 55(8):22852294. https://doi.org/10.1007/s00125-012-2564-7

12. Toivonen RK, Emani R, Munukka E et al (2014) Fermentable fibres condition colon microbiota and promote diabetogenesis in NOD mice. Diabetologia 57(10):2183-2192. https://doi.org/10.1007/ s00125-014-3325-6

13. Xiao L, Van't Land B, Engen PA et al (2018) Human milk oligosaccharides protect against the development of autoimmune diabetes in NOD-mice. Sci Rep 8(1):3829. https://doi.org/10.1038/ s41598-018-22052-y

14. Aachary AA, Prapulla SG (2011) Xylooligosaccharides (XOS) as an emerging prebiotic: microbial synthesis, utilization, structural characterization, bioactive properties, and applications. Compr Rev Food Sci Food Saf 10:1-16

15. Nicklas W, Deeny A, Diercks P, Gobbi A, Illgen-Wilcke B, Seidelin M (2010) FELASA guidelines for the accreditation of health monitoring programs and testing laboratories involved in health monitoring. Lab Anim (NY) 39(2):43-48. https://doi.org/10.1038/ laban0210-43

16. Hansen CH, Yurkovetskiy LA, Chervonsky AV (2016) Cutting edge: commensal microbiota has disparate effects on manifestations of polyglandular autoimmune inflammation. J Immunol 197(3): 701-705. https://doi.org/10.4049/jimmunol.1502465

17. Canibe N, Hojberg O, Badsberg JH, Jensen BB (2007) Effect of feeding fermented liquid feed and fermented grain on gastrointestinal ecology and growth performance in piglets. J Anim Sci 85(11): 2959-2971. https://doi.org/10.2527/jas.2006-744

18. Poulsen F, Jensen KB (2007) A luminescent oxygen channeling immunoassay for the determination of insulin in human plasma. J Biomol Screen 12(2):240-247. https://doi.org/10.1177/ 1087057106297566

19. Pridal L, Ingwersen SH, Larsen FS, Holst JJ, Adelhorst K, Kirk O (1995) Comparison of sandwich enzyme-linked immunoadsorbent assay and radioimmunoassay for determination of exogenous glucagon-like peptide-1(7-36)amide in plasma. J Pharm Biomed Anal 13(7):841-850. https://doi.org/10.1016/0731-7085(95) 01312-9

20. Jablonski KA, Amici SA, Webb LM et al (2015) Novel markers to delineate murine M1 and M2 macrophages. PLoS One 10(12): e0145342. https://doi.org/10.1371/journal.pone.0145342

21. De VF, Kovatcheva-Datchary P, Goncalves D et al (2014) Microbiota-generated metabolites promote metabolic benefits via gut-brain neural circuits. Cell 156:84-96

22. Hansen CH, Krych L, Buschard K et al (2014) A maternal glutenfree diet reduces inflammation and diabetes incidence in the offspring of NOD mice. Diabetes 63(8):2821-2832. https://doi.org/10. 2337/db13-1612

23. Antvorskov JC, Josefsen K, Haupt-Jorgensen M, Fundova P, Funda DP, Buschard K (2016) Gluten-free diet only during pregnancy efficiently prevents diabetes in NOD mouse offspring. J Diabetes Res 2016:3047574
24. Dall M, Calloe K, Haupt-Jorgensen M et al (2013) Gliadin fragments and a specific gliadin 33-mer peptide close KATP channels and induce insulin secretion in INS-1E cells and rat islets of langerhans. PLoS One 8(6):e66474. https://doi.org/10.1371/ journal.pone. 0066474

25. Haupt-Jorgensen M, Buschard K, Hansen AK, Josefsen K, Antvorskov JC (2016) Gluten-free diet increases beta-cell volume and improves glucose tolerance in an animal model of type 2 diabetes. Diabetes Metab Res Rev 32(7):675-684. https://doi.org/10. 1002/dmrr.2802

26. Meslin JC, Andrieux C, Sakata T et al (1993) Effects of galactooligosaccharide and bacterial status on mucin distribution in mucosa and on large intestine fermentation in rats. Br J Nutr 69(3):903912. https://doi.org/10.1079/BJN19930090

27. Sapone A, de ML, Pietzak M et al (2006) Zonulin upregulation is associated with increased gut permeability in subjects with type 1 diabetes and their relatives. Diabetes 55(5):1443-1449. https://doi. org/10.2337/db05-1593

28. Burrows MP, Volchkov P, Kobayashi KS, Chervonsky AV (2015) Microbiota regulates type 1 diabetes through Toll-like receptors. Proc Natl Acad Sci U S A 112(32):9973-9977. https://doi.org/10. 1073/pnas.1508740112

29. Yurkovetskiy L, Burrows M, Khan AA et al (2013) Gender bias in autoimmunity is influenced by microbiota. Immunity 39(2):400 412. https://doi.org/10.1016/j.immuni.2013.08.013

30. $\mathrm{Hu} \mathrm{Y}$, Peng J, Tai $\mathrm{N}$ et al (2015) Maternal antibiotic treatment protects offspring from diabetes development in nonobese diabetic mice by generation of tolerogenic APCs. J Immunol 195(9):41764184. https://doi.org/10.4049/jimmunol.1500884

31. Vazquez E, Santos-Fandila A, Buck R, Rueda R, Ramirez M (2017) Major human milk oligosaccharides are absorbed into the systemic circulation after oral administration in rats. Br J Nutr 117(2):237247. https://doi.org/10.1017/S0007114516004554

32. Eiwegger T, Stahl B, Haidl P et al (2010) Prebiotic oligosaccharides: in vitro evidence for gastrointestinal epithelial transfer and immunomodulatory properties. Pediatr Allergy Immunol 21(8): 1179-1188. https://doi.org/10.1111/j.1399-3038.2010.01062.x

33. Naarding MA, Ludwig IS, Groot F et al (2005) Lewis X component in human milk binds DC-SIGN and inhibits HIV-1 transfer to CD4+ T lymphocytes. J Clin Invest 115(11):3256-3264. https:// doi.org/10.1172/JCI25105

34. Hanninen A, Toivonen R, Poysti S et al (2017) Akkermansia muciniphila induces gut microbiota remodelling and controls islet autoimmunity in NOD mice. Gut 67:1445-1453

35. Kverka M, Zakostelska Z, Klimesova K et al (2011) Oral administration of Parabacteroides distasonis antigens attenuates experimental murine colitis through modulation of immunity and microbiota composition. Clin Exp Immunol 163(2):250-259. https://doi. org/10.1111/j.1365-2249.2010.04286.x

36. Vaarala O, Atkinson MA, Neu J (2008) The 'perfect storm' for type 1 diabetes: the complex interplay between intestinal microbiota, gut permeability, and mucosal immunity. Diabetes 57(10):2555-2562. https://doi.org/10.2337/db08-0331

37. Meddings JB, Jarand J, Urbanski SJ, Hardin J, Gall DG (1999) Increased gastrointestinal permeability is an early lesion in the spontaneously diabetic BB rat. Am J Physiol 276(4):G951-G957. https://doi.org/10.1152/ajpgi.1999.276.4.G951

38. Neu J, Reverte CM, Mackey AD et al (2005) Changes in intestinal morphology and permeability in the biobreeding rat before the onset of type 1 diabetes. J Pediatr Gastroenterol Nutr 40(5):589-595. https://doi.org/10.1097/01.MPG.0000159636.19346.C1

39. McCullogh JS, Ratcliffe B, Mandir N, Carr KE, Goodlad RA (1998) Dietary fibre and intestinal microflora: effects on intestinal 
morphometry and crypt branching. Gut 42(6):799-806. https://doi. org/10.1136/gut.42.6.799

40. Hadjiyanni I, Li KK, Drucker DJ (2009) Glucagon-like peptide-2 reduces intestinal permeability but does not modify the onset of type 1 diabetes in the nonobese diabetic mouse. Endocrinology 150(2):592-599. https://doi.org/10.1210/en.2008-1228

41. Lee AS, Gibson DL, Zhang Y, Sham HP, Vallance BA, Dutz JP (2010) Gut barrier disruption by an enteric bacterial pathogen accelerates insulitis in NOD mice. Diabetologia 53(4):741-748. https://doi.org/10.1007/s00125-009-1626-y

42. Bosi E, Molteni L, Radaelli MG et al (2006) Increased intestinal permeability precedes clinical onset of type 1 diabetes. Diabetologia 49(12):2824-2827. https://doi.org/10.1007/s00125006-0465-3
43. Carratu R, Secondulfo M, de ML et al (1999) Altered intestinal permeability to mannitol in diabetes mellitus type I. J Pediatr Gastroenterol Nutr 28(3):264-269. https://doi.org/10.1097/ 00005176-199903000-00010

Publisher's note Springer Nature remains neutral with regard to jurisdictional claims in published maps and institutional affiliations. 